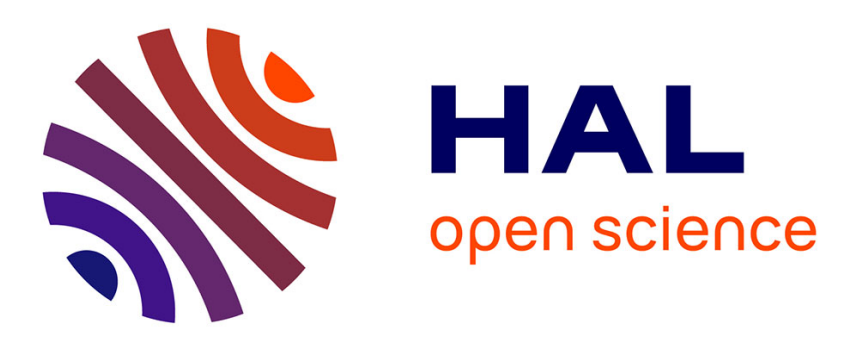

\title{
L'harmonisation du classement des bois bruts dans la Communauté Economique Européenne
}

\author{
Xavier Le Chatelier
}

\section{To cite this version:}

Xavier Le Chatelier. L'harmonisation du classement des bois bruts dans la Communauté Economique Européenne. Revue forestière française, 1965, 4, pp.257-263. 10.4267/2042/24726 . hal-03389846

\section{HAL Id: hal-03389846 https://hal.science/hal-03389846}

Submitted on 21 Oct 2021

HAL is a multi-disciplinary open access archive for the deposit and dissemination of scientific research documents, whether they are published or not. The documents may come from teaching and research institutions in France or abroad, or from public or private research centers.
L'archive ouverte pluridisciplinaire HAL, est destinée au dépôt et à la diffusion de documents scientifiques de niveau recherche, publiés ou non, émanant des établissements d'enseignement et de recherche français ou étrangers, des laboratoires publics ou privés. 


\section{REVUE FORESTIERE FRANÇAISE}

1965

AVRIL

No 4

Dans ce numéro: $\mathrm{X}$. LE Chatelier: L'harmonisation du classement des bois bruts dans la Communauté Economique Européenne. - J. SonNIER: L'aménagement des séries domaniales de restauration des Basses-Alpes occidentales. - G. Soulères: Essai de régénération artificielle en chêne pédonculé en forêt d'Amont-Aval (Bresse Comtoise). - C. BARToli: La mystique de la «forêt-climax » et la mystique de « l'usine à bois 》.

\section{L'HARMONISATION DU CLASSEMENT DES BOIS BRUTS DANS LA COMMUNAUTE ECONOMIQUE EUROPEEENNE}

PAR

Xavier LE CHATELIER

Chef de la division « forêts et produits forestiers 》

Direction Générale de l'Agriculture

Commission de la C.E.E.

Bruxelles

Le classement des bois bruts a pour but de caractériser les produits de l'exploitation forestière selon des critères que l'on peut mesurer ou apprécier. Il conduit à la formation de classes et à une certaine normalisation des caractéristiques de chaque classe; il est donc en relation directe avec la formation du prix.

Tant que le bois ne fait l'objet que d'échanges locaux, c'est-àdire dans une économie de subsistance, le classement reste une affaire locale; il est adapté aux particularités de l'utilisation et aux essences existant dans la région même. On conçoit aisément qu'un système de classement entièrement différent puisse exister dans une région voisine et c'est ainsi que divers systèmes ont pu coexister et coexistent encore dans un même pays.

Mais, avec l'avènement et le développement de l'économie de marché pour les produits de l'exploitation forestière, le commerce élargit progressivement son rayon d'action à l'échelon du pays entier d'abord, puis, au delà des frontières, sur le plan international. Alors, comme dans les autres secteurs de l'économie en pareil cas, la nécessité d'une certaine normalisation se fait sentir. Certains 
pays ont ainsi été amenés à instituer des classements officiels qui sont appliqués sur l'ensemble de leur territoire: c'est le cas de la HOMA (1) par exemple qui existe en Allemagne depuis 1936.

Mais le commerce international du bois s'est considérablement développé en Europe depuis la fin de la deuxième Guerre Mondiale et l'on a souvent mentionné dans diverses instances l'intérêt que présenterait un classement international des bois pour faciliter ce commerce. Il convient en effet de signaler que les classements actuellement en usage dans les divers pays présentent une diversité déconcertante, ne serait-ce qu'en raison des unités de mesure du bois utilisées dans les pays anglo-saxons.

Cependant, en vue d'unifier les classements de plusieurs pays, certaines tentatives récentes ont été faites parmi lesquelles on doit citer l'initiative de la $5^{\mathrm{e}}$ Commission (Forêt privée et communale) de la Confédération Européenne de l'Agriculture: des travaux entrepris en 1958 ont abouti en 1961 à l'élaboration par un groupe d'experts de six pays européens sous la direction de M. H.G. WiNKELMANN (Suisse) de directives pour un classement européen uniforme des bois (2).

La Communauté Economique Européenne doit constituer dès la fin de la période de transition, le $1^{\text {er }}$ janvier 1970, un espace économique unique. Déjà, pour le bois brut, il n'y a plus aucune restriction quantitative au commerce intracommunautaire. La liberté d'établissement, la libre prestation des services, la libre circulation des travailleurs et des capitaux entre les six pays du marché commun doit tout naturellement entraîner une intensification des échanges de bois notamment dans les régions frontières - et les régions frontières de l'est de la France et des zones limitrophes sont particulièrement boisées. Déjà, des entreprises industrielles s'approvisionnent en bois matière première indistinctement des deux côtés de la frontière. Il n'est pas douteux que des règles communes de classement faciliteraient ces échanges intracommunautaires: l'acheteur et le vendeur parlant le même langage pourraient ainsi définir sans ambiguité un lot de bois déterminé.

Par ailleurs, dans le cadre des études nécessaires à la coordination des politiques forestières suivies par les Etats membres de la C.E.E., il importe de pouvoir disposer d'une statistique forestière communautaire suffisamment précise et détaillée. A cet égard, un classement commun apparaît comme un instrument indispen-

(1) HOMA: Holzmessanweisung (Bestimmungen über die Ausformung, Messung und Sortenbildung des Holzes in den deutschen Forsten).

(2) Confédération Européenne de l'Agriculture (C.E.A.). Directives pour un classement européen uniforme des bois. Brougg, Suisse. 1961. Publications de la CEA, fascicule 19. 
sable pour comparer sur une base rigoureuse les données des six pays relatives à' la production, au commerce, à la consommation, aux prix des produits forestiers.

Indiquons enfin comment se présente actuellement la situation au point de vue du classement des bois dans les six pays du marché commun:

En République Fédérale d'Allemagne, il existe un système de classement officiel, la HOMA dont il a été question plus haut, institué par la loi en 1936 et applicable obligatoirement à tous les propriétaires de forêts. Ce système, déjà âgé de près de. 30 ans, n'est plus parfaitement adapté aux conditions actuelles et devrait être modifié et assoupli.

En France, il n'existe pas de législation mais seulement des recommandations générales réglant les usages en ce qui concerne' le bois façonné. Cependant, dans les trois départements de l'Est où l'exploitation se fait en régie, il existe un classement administratif qui est un règlement intérieur à l'administration.

En Italie, comme en France, il n'y a pas de loi, mais seulement des' usages qui varient selon les provinces.

En Belgique, la situation se rapproche de la France en pratique, bien qu'une codification non obligatoire ait été publiée en 1936.

Au Grand-Duché de Luxembourg, un système très proche de la HOMA. allemande est en usage, mais n'a pas force de loi; il est cependant appliqué strictement par l'Etat et pratiquement par l'ensemble des propriétaires forestiers.

Aux Pays-Bas enfin, des directives pour la classement et le mesurage des bois indigènes, traitant des bois sur pied et des bois abattus, ont été publiées en 1960. Elles ne sont pas obligatoires, mais appliquées sur une base volontaire par la grande majorité des propriétaires et des exploitants.

A $\mathrm{A}$, demande des chefs des administrations forestières des six pays, la Commission a réuni en octobre 1962 un groupe d'experts gouvernementaux pour étudier ce problème et envisager l'harmonisation de ces usages et réglementations, Ce groupe d'experts a élaboré aú cours de six réunions de travail un avant-projet de (" directive sur le mesurage et le classement des bois bruts " "sür lequel un accord non sans réserves a été atteint. It est certain que tout travail d'élaboration d'une réglêmentation communaútaire sou- 
lève de graves difficultés car il est rare qu'un pays consente de bon gré à modifier sa réglementation ou ses habitudes. Contrairement aux Organisations internationales dont les recommandations ou décisions peuvent rester parfois lettre morte, la Communauté Economique Européenne est une organisation supranationale dont l'activité doit se traduire par des faits dans les Etats membres, d'où la passion que soulèvent parfois les discussions.

Il est apparu dès le début des travaux qu'il était nécessaire de se limiter au classement des bois bruts façonnés, pour lesquels on peut mesurer ou apprécier facilement la grosseur et les qualités. Il ne saurait être question de classer facilement et rigoureusement les bois sur pied en raison des erreurs inévitables d'appréciation que comporterait une telle opération. Il est naturel que les pays où se pratique généralement la vente sur pied, la France et la Belgique, paraissent moins intéressés par un classement ocmmun que les pays qui vendent leurs bois abattus et façonnés. Cependant, même pour la France et la Belgique, le classement des bois façonnés présente un intérêt certain: s'il n'intervient pas pour la fixation du prix des bois sur pied, il intéresse, non pas le propriétaire lui-même, mais l'exploitant qui doit façonner ses bois en fonction des débouchés.

Une question de principe s'est posée aux experts : fallait-il adopter un classement absolu ou un classement par destination? Le classement absolu ne fait intervenir que la grosseur et éventuellement la qualité indépendamment de l'usage auquel on destine le bois. Au contraire, un classement par destination prend en considération les desiderata de l'utilisateur et aboutit ainsi à des assortiments dénommés " bois de déroulage et de tranchage ", " pilots » ou " bois de trituration » par exemple. Les deux procédés ont leur intérêt (et peuvent d'ailleurs être combinés); le classement absolu n'est pas lié à l'emploi; il s'adresse à un éventail plus large d'acheteurs, donc à un marché plus étendu, mais il est relativement grossier. Le classement par utilisation est mieux à même de satisfaire une catégorie d'acheteurs bien déterminée; c'est un classement plus fin, plus soigneux, par conséquent plus coûteux, mais qui devrait de ce fait permettre d'obtenir un meilleur prix du bois. On a cependant estimé que, devant l'évolution rapide et la diversification des emplois du bois de nos jours, un classement par utilisation risquait d'être rapidement démodé et qu'il convenait d'adopter un classement de type absolu, quitte à le compléter éventuellement par des " assortiments spéciaux » (classés par utilisation).

On a enfin convenu d'employer le mot bois bruts, et non bois ronds généralement utilisé en France, parce que ce terme a paru plus précis et qu'il est déjà utilisé dans un texte officiel, la Nomenclature douanière de Bruxelles. 


\begin{abstract}
***
L'avant-projet de directive doit maintenant subir la procédure d'approbation par la Commission d'abord puis par le Conseil et peut donc être encore largement amendé ou modifié; il ne saurait être question de l'exposer ici en détail dès maintenant. On peut cependant en donner une rapide description et mettre l'accent pour le lecteur français sur les points susceptibles de l'intéresser.

Ce projet se présente sous forme de la directive proprement dite qui définit le cadre juridique et la nature des obligations faites aux Etats membres, et d'une annexe qui précise les modalités des opérations de mesurage et de classement.
\end{abstract}

\title{
1. - Directive proprement dite
}

Ce projet de texte juridique ëst conçu de façon très souple. Chaque pays doit ouvrir des possibilités légales pour l'application du classement commun mais reste libre d'en fixer lui-même le champ d'application; c'est dire que chaque pays peut, s'il le désire, rendre tout ou partie du classement obligatoire à l'ensemble ce sa production forestière, ou à certains produits seulement, tels que les grumes résineuses, ou encore à certaines forêts (forêts domaniales par exemple). Chaque Etat membre doit en outre prescrire que les bois bruts, lors de la commercialisation, ne peuvent être désignés en tant que bois bruts classés CEE que s'ils ont été classés selon l'annexe. En pratique, il est vraisemblable que ce classement restera d'application facultative dans tous les pays, même en Allemagne; on a constaté en effet dans ce pays qu'un classement obligatoire impose une rigidité souvent incompatible avec les nécessités pratiques.

Le projet prévoit également la possibilité pour les pays de détailler le classement prévı par l'annexe.

\section{2. - Annexe}

Cette annexe qui constitue la partie technique du projet de directive comporte deux parties ayant trait l'une au mesurage, l'autre au classement.

C'est à propos du mesurage que de longues discussions ont opposé les experts français et belges à ceux des autres pays. La France et la Belgique ont en effet adopté depuis un certain nombre d'années et répandu dans les usages le principe de la mesure de la circonférence des grumes alors que les autres pays de la Communauté et la plupart des pays au monde emploient la mesure dı diamètre. Les discussions aut sein du groupe de travail ont donné lieu à de brillants exposés des tenants des deux systèmes, d'où il ressort que ces deux procédés sont pratiquement équivalents. En déf- 
nitive, il a été admis, malgré les réticences des pays intéressés, que la mesure du diamètre était préférable parce que c'était le procédé le plus répandu. Les experts français ont pris l'engagement de défendre cette thèse auprès de l'AFNOR et des organisations professionnelles en faisant valoir que la création d'unę communauté économique, c'est-à-dire d'un espace économique unique, entraîne nécessairement dans ce secteur comme dans d'autres la modification de certains usages nationaux.

Quant aux autres dispositions prévues pour le mesurage, elles ne présentent aucune différence de principe avec les usages français actuels.

En ce qui concerne le classement proprement dit, le projet évite à dessein des prescriptions trop détaillées sur lesquelles un accord aurait été difficile à obtenir, et se limite à tracer un cadre général que les pays pourront détailler à volonté. Ce cadre s'inspire dans une large mesure, pour les grumes tout au moins, des propositions de la CEA et des règles en usage en Allemagne, au Luxembourg, aux Pays-Bas ainsi que dans un certain nombre de pays européens; des classes de dimension sont établies de 10 en 10 centimètres de diamètre au milieu sous écorce avec possibilité de subdiviser chaque classe. Pour les perches, c'est le diamètre sur écorce à 1 mètre du gros bout qui est pris en considération; pour les bois en stères, c'est le diamètre sur écorce au fin bout.

Quant au classement par qualité, les experts français avaient suggéré que l'on introduise dans la définition des classes de qualité A. $B$ et $C$ des précisions chiffrées pour certains critères mesurables, tels que la courbure, la décroissance, le fil tors, le méplat.... ce procédé paraissant particulièrement souhaitable pour les feuillus. Les discussions ont malheureusement montré qu'il était impossible de s'entendre sur de telles normes chiffrées, car ces normes varient essentiellement selon l'emploi: certaines tolérances sont admises par exemple pour les grumes à sciage et non pour les poteaux et pilots, et inversement. On a dû se contenter de définitions de classes de qualité forcément imprécises en envisageant néanmoins de définir ultérieurement des classes plus précises pour des assortiments bien déterminés.

\section{3. - Remarque}

Comme on le voit, le projet de classement rapidement décrit cidessus est essentiellement un classement de type absolu. Il avait été envisagé d'abord de le compléter par un classement par destination présumée au moins pour un petit nombre d'assortiments commercialisés sur une large échelle, tels que le bois de tranchage et le déroulage, le bois de mine, le bois de trituration. Là encore, des difficultés insurmontables ont été rencontrées, et l'on a estimé qu'à 
moins de se contenter d'une énumération sans portée pratique, il valait mieux n'en pas parler.

Rappelons que cette directive n'est encore qu'au stade de l'avantprojet et qu'une longue procédure doit intervenir avant son adoption définitive. Elle n'est pas parfaite; son application aux résineux ne présentera généralement pas de difficultés mais le classement des feuillus posera encore longtemps des problèmes ardus. Cependant une telle initiative est nécessaire; elle doit être considérée comme un premier stade dans une évolution souhaitable. Ses dispositions n'étant pas contraignantes, elle ne peut apporter de gêne aux pays, mais elle ne devra pas rester lettre morte. C'est par adhésion volontaire qu'elle devra entrer dans les habitudes; les pays devront en préconiser l'adoption par les milieux intéressés tout en s'efforçant de s'y conformer rapidement dans le domaine soumis au régime forestier. L'expérience de l'adhésion volontaire à un classement non obligatoire a déjà fait ses preuves dans d'autres pays. Il est d'ailleurs probable que l'accroissement des échanges intracommunautaires de bois bruts favorisera naturellement la mise en pratique de ces dispositions.

Enfin, ainsi qu'il a été dit plus haut, un classement européen uniforme des bois préoccupe depuis plusieurs années les milieux forestiers d'un certain nombre de pays qui suivent attentivement les travaux qui se poursuivent au sein de la CEE: il est vraisemblable que, le jour où ces travaux auront abouti, la directive CEE fera tache d'huile et que d'autres pays s'efforceront d'adopter un système analogue sinon identique. 